\title{
Dr. Kiosk
}

\author{
Mohammed Ajmal K.B ${ }^{1}$, Mohammed Fayaz N.H ${ }^{2}$, Pavin Krishna ${ }^{3}$, Muneebah Mohyiddeen ${ }^{4}$ \\ B. Tech Student, Department of Computer Engineering, Universal Engineering College, Vallivattom, Kerala, India ${ }^{1,2,3}$ \\ Assistant Professor, Department of Computer Engineering, Universal Engineering College, Vallivattom, Kerala, India ${ }^{4}$
}

\begin{abstract}
The long queue in hospital often causes delaying in availability of services, Delayed diagnosis and other diagnostic errors are more common, costly and harmful than any other patient safety threat overall are the 6th leading cause of death in the United States(Report by NCBI),The DR.KIOSK is virtual Doctor Kiosk where a person could get diagnosed with virtual presence of a Doctor with AI assistance, which means same quality treatment available in hospital will be available inside a cubicle that too without having a physical presence of your doctor with intelligent system for reducing diagnosing errors, DR KIOSK is similar to Auto Teller Machine where you can meet your Doctor Online through this kiosk which has equipment equivalent to stethoscope etc ,this diagnostic reports is sent to Doctor and his prescription is Broadcast to group of nearby pharmacist literally patient doesn't need to bother about long queues both in pharmacy and Clinic
\end{abstract}

Keywords: Internet of things; Global system for mobile communication; PIR sensor; Raspberry Pi 3 model B

\section{INTRODUCTION}

According to a survey conducted by NCBI "Delayed diagnosis and other diagnostic errors are more common, costly and harmful than any other patient safety threat overall are the 6th leading cause of death another challenge is to cater to the deserving undeserved community of Tier 4 locations and below which need due attention. Public health services have done poorly even along the income distribution dimension. According to a study, the poorest $20 \%$ of the population captures only $10 \%$ of the public health subsidy as compared to $30 \%$ by the richest $20 \%$. The share in the subsidy rises monotonically as we move from the bottom $20 \%$. The discrepancy and poor implementation of budget allocation have left a stark gap in rural and urban health care services. With India ascending the health care services on the tertiary level and becoming the new face of global medical tourism, it dampens the zeal to notice the staggering figure of major gamut being unable to access affordable primary health care services which are necessary for the development of the nation. The helmets like Atttapady in Kerala or Muttur in Karnataka are one of the poor villages in India neither have proper facility for medical treatment nor they keep a personal medical data. People need to migrate from these areas to have a proper medical checkup which will cost their year's hardship and it is impossible for them to raise such kind of amount. This cause high death rate in these area As we researched we also found that India we doesn't have centralized medical data we're you can access your medical reports anywhere in India and there is no system to rate Doctors. Which cause errors in medical diagnostics and which is another major problem we're facing in India. The scope of the problem is very large, here we're addressing a worldwide problem with huge number of concerns and problem, by giving medical assistance to the people in helmets in India with world class quality with no medical errors, and this is ensured by Dr.Kiosk by using leading technologies to solve a world-wide problem To solve this problems we introduce Dr.Kiosk which look-like ATM kiosk which will be having a LCD screen and all necessary equipment to check BP, Heart-rate for the primary medical analysis, purpose of this project is to reduce delayed diagnosing by placing this where ever it needs to be in and to address the second biggest problem that is medical errors we use power of machine learning to give predictions to the doctor and Dr.Kiosk will also records medical reports where you can access from it from anywhere in the World using your Bio-metrics or user id and password which you will get at the time of the registration .

\subsection{Scope of project}

The challenge is to cater to the deserving under-served community of Tier 4 locations and below which need due attention. Public health services have done purely even along the income distribution dimension. According to a study, the poorest $20 \%$ of the population capture only $10 \%$ of the public health subsidy as compared to $30 \%$ by the richest $20 \%$. The share in the subsidy rises monotonically as we move from the bottom $20 \%$. The discrepancy and poor implementation of budget allocation have left a stark gap in rural and urban health care services. With India ascending the health care services on the tertiary level and becoming the new face of global medical tourism, it dampens the zeal to notice the staggering figure of major gamut being enable to access affordable primary health care services which are necessary for the development of the nation. 
Vol. 8, Issue 4, April 2019

\subsection{Problem Statement}

The long queue in hospital often causes delaying in availability of services, Delayed diagnosis and other diagnostic errors are more common, costly and harmful than any other patient safety threat overall are the 6th leading cause of death in the United States(Report by NCBI),The DR.KIOSK is virtual Doctor Kiosk where a person could get diagnosed with virtual presence of a Doctor with AI assistance, which means same quality treatment available in hospital will be available inside a cubicle that too without having a physical presence of your doctor with intelligent system for reducing diagnosing errors, DR KIOSK is similar to Auto Teller Machine where you can meet your Doctor Online through this kiosk which has equipment equivalent to stethoscope etc ,this diagnostic reports is sent to Doctor and his prescription is Broadcast to group of nearby pharmacist literally patient doesn't need to bother about long queues both in pharmacy and Clinic

\section{LITERATURE SURVEY}

In $^{[1]}$ developed a novel priority-setting method for patient safety problems and solutions called PRIORITIZE. Over more than $500 \mathrm{NW}$ London clinicians via an open-ended questionnaire to identify three main problems and solutions relating to delayed diagnosis in primary care. 113 clinicians submitted their suggestions which were thematically grouped and synthesized into a composite list of 33 distinct problems and 27 solutions. A random group of 75 clinicians from the initial cohort scored these and an overall ranking was derived. The agreement between the clinicians' scores was presented using the Average Expert Agreement, adopted a definition of delayed diagnosis as "a diagnosis that was unintentionally delayed while sufficient information was available earlier",and developed PRIORITIZE method, by modifying the Child Health and Nutrition Research Initiative (CHNRI) methodology for patient safety context to determine the main problems and solutions relating to delayed diagnosis in primary care.

The method utilizes participants' perspectives to customize a priority agenda based on the local context and needs. The CHNRI methodology has been used widely to inform policy makers, funder and international organizations about research gaps and resource priorities. Project steering group determined the scope, the focus, the context and the criteria of the priority setting exercise, A survey was sent out to the clinicians inviting them to identify priorities based on the requirements and information set out by the project steering group, Clinicians' suggestions were refined and collated into a composite set of priorities, Clinicians were invited to score the composite set of the priorities they suggested according to the criteria established by the project steering group, The project steering group was provided with a final ranked list of priorities based on clinicians' perspective, Final ranked list of priorities guided the Patient Safety Board in shaping a list of actions and timeline for those as well as their wider dissemination back to clinicians and other stakeholders. While the CHNRI methodology invites experts in the relevant field to nominate research priorities, PRIORITIZE focused on priorities in healthcare services delivery and identified clinicians' as experts. The PRIORITIZE methodology determined priorities by focusing on the topic from two complementary angles: problems and solutions.

The final output of this approach is presenting the top priorities categorized according to the level at which these could be implemented:

a) actions for general practitioners;

b) actions for general practice organizations; and

c) actions for health system custodians.

This study was deemed to be a service evaluation and quality and safety improvement initiative and consequently did not require ethics or research governance approval according to the UK's Health Research Authority guidance. During the study's first stage, the project steering group (Imperial College Health Partners) considered previous evidence on patient safety in primary care in the UK and decided to focus on medication safety (presented elsewhere) and delayed diagnosis. They also chose the criteria guiding prioritization of collated suggestions, i.e. scoring of problems and solutions

$\mathrm{In}^{[2]}$ Diagnostic errors and abnormal diagnostic tests lost to follow-up: a source of needless waste and delay to treatment, Diagnostic errors are an important and often appreciate source of medical error, needless delays to treatment, and needlessly wasted resources. Almost $65 \%$ of diagnostic errors have an important contribution of system errors, of which many are an abnormal test result that was lost to follow-up, that is, missed results. These system problems that contribute to missed results may represent low-hanging fruit for those who wish to reduce diagnostic errors in their institution. The rate of missed results and associated treatment delay are discussed. The system factors and human factors that contribute to these errors are discussed along with strategies that can be adopted to reduce these errors with treatment delays. The most common $\mathrm{In}^{[3]}$ Diagnostic errors associated with the failure to follow up on abnormal diagnostic studies ("missed results") are a potential cause of treatment delay and a threat to patient safety. Few data exist concerning the frequency of missed results and associated treatment delays within the Veterans Health Administration (VA). The primary objective of the study was to assess the frequency of missed results and resulting treatment delays encountered by primary care providers in VA clinics. An anonymous on-line survey of primary care 


\author{
Vol. 8, Issue 4, April 2019
}

providers was conducted as part of the health systems ongoing quality improvement programs. They collected information from providers concerning their clinical effort (e.g., number of clinic sessions, number of patient visits per session), number of patients with missed abnormal test results, and the number and types of treatment delays providers encountered during the two week period prior to administration of our survey.

The survey was completed by 106 out of 198 providers ( 54 percent response rate). Respondents saw and average of 86 patients per 2 week period. Providers encountered 64 patients with missed results during the two week period leading up to the study and 52 patients missed results included imaging studies ( 29 percent), clinical laboratory ( 22 percent), anatomic pathology (9 percent), and other (40 percent). The most common diagnostic delays were cancer (34 percent), endocrine problems (26 percent), cardiac problems (16 percent), and others (24 percent). Missed results leading to clinically important treatment delays are an important and likely under appreciated source of diagnostic error.

$\mathrm{In}^{[4]}$ Missed and delayed diagnoses in the ambulatory setting: a study of closed malpractice claims. Although missed and delayed diagnoses have become an important patient safety concern, they remain largely unstudied, especially in the outpatient setting. developed a framework for investigating missed and delayed diagnoses, advance understanding of their causes, and identify opportunities for prevention. Retrospective review of 307 closed malpractice claims in which patients alleged a missed or delayed diagnosis in the ambulatory setting. Diagnostic errors associated with adverse outcomes for patients, process breakdowns, and contributing factors.

A total of 181 claims (59\%) involved diagnostic errors that harmed patients. Fifty-nine percent (106 of 181$)$ of these errors were associated with serious harm, and 30\% (55 of 181) resulted in death. For 59\% (106 of 181) of the errors, cancer was the diagnosis involved, chiefly breast (44 claims [24\%]) and colorectal (13 claims [7\%]) cancer. The most common breakdowns in the diagnostic process were failure to order an appropriate diagnostic test (100 of 181 [55\%]), failure to create a proper follow-up plan (81 of 181 [45\%]), failure to obtain an adequate history or perform an adequate physical examination (76 of 181 [42\%]), and incorrect interpretation of diagnostic tests (67 of 181 [37\%]). The leading factors that contributed to the errors were failures in judgment (143 of 181 [79\%]), vigilance or memory (106 of 181 [59\%]), knowledge (86 of 181 [48\%]), patient-related factors (84 of 181 [46\%]), and handoffs (36 of 181 [20\%]). The median number of process breakdowns and contributing factors per error was 3 for both (interquartile range, 2 to 4 ).

Reviewers were not blinded to the litigation outcomes, and the reliability of the error determination was moderate. Diagnostic errors that harm patients are typically the result of multiple breakdowns and individual and system factors. Awareness of the most common types of breakdowns and factors could help efforts to identify and prioritize strategies to prevent diagnostic errors.

Waiting time as the period of time an individual wait until a specific action occurs. Waiting time was also defined as the time a patient entered a clinic or service point and gets consulted by a doctor with a prescription in his hand. There are two different ways in which the waiting time has been defined in the past. At first, waiting time is the time that starts when an individual comes for the appointment and it continues until the individual gets consulted by the doctor. In the second definition, it starts when an individual comes for taking appointment and ends when he got prescribed medicine after being consulted by the doctor. With the passage of time, long waiting lines at doctor's clinics became a major problem in developing countries.

$\mathrm{In}^{[5]}$ a South African health clinic, block appointment system was introduced as an experiment, in which waiting time for patients was measured for the period of one week before and after the implementation of the appointment system. During the experiment, interviews were taken from focus-group, staff, and patients and from results it was realized that acute medically ill patients with prior appointments had short waiting times as compared to the patients without appointments. It has also been stated that the appointment system has no benefit for the patients not seeing the doctor or who are taking routine repeated medication. Later it was realized that the block appointment system offers shorter waiting time only for acutely ill patients and not for others.

$\mathrm{In}^{[6]}$ It has been proved in the past research that there is a direct relationship between appointment delay and cancellation of the appointment. Appointment delay is defined as the time which starts when an individual request for an appointment and ends until he/ she gets consulted by the doctor. Longer appointment delays cause more appointment 10 cancellations. So, the best way to reduce appointment cancellations or no-shows is to minimize the time gap between the appointment request and doctor's checkup/consultation time. Minimizing this gap is called open access (abbreviated as OA) or advance access policy which later became a popular practice and part of an active research also. Both positive and negative results were experienced by the researchers in their experiments. Some practitioners were in the favour of OA and highly recommended it, and on the other hand, there were some practitioners who disagreed and were against of OA implementation.

$\mathrm{In}^{[7]} \mathrm{a}$ health care centre, an application which is used to manage and minimize patient waiting time is called managing patient's appointment system. Some health care clinics use this kind of applications while some do not. Medical clinics who use such applications usually have shorter waiting times as compared to those medical clinics who do not use any appointment application. Patients who supposed to wait more than an hour for their medical check-up feel disgraced and unfair. Patients can evaluate the quality of any medical centre based on the waiting time they have to experience. Therefore, it has become important to consider factors like "saving time" and "minimizing idle time" while developing any patient's appointment system. Whereas according to Klassen (2002), scheduling doctor's time and patient's appointment supposed to depend on the seriousness of the case and patient's need. This is a critical issue, especially in 
an industry that increasingly views patients as consumers. "People need to understand that patients are still customers," seasoned call center consultant Irene Vergules told PatientEngagementHIT.com in a past interview. "We need to really look at what our access looks like to our providers." As patients shop around for convenient care options, health care organizations need to meet those demands.

$\mathrm{In}^{[8]} \mathrm{An}$ online appointment system is a web-based system which is made up of independent components or web pages, working together for a common purpose. Such systems are available on the internet for the users to accomplish some purpose. Internet as a system which provides an opportunity to millions of people to get access to a large amount of stored data and get connected to each other despite distances. Increased rate of missed appointments and patient dissatisfaction pushed to recognize the need for better-quality care services. With the advancement of information technology and the sensitive situation of medical care, online appointment system emerged as an important output for efficient and timely delivery of health care services. Earlier, appointment requests were used to make through the phone call, email or fax but with the advancement of technology and internet, the trend changed towards the proper online appointment systems. According to Gruca (2004), a patient can make an appointment for a doctor either by going directly to the doctor's clinic or making a manual appointment through the phone call or sending the email. With the emergence of the internet, health care centre can connect and communicate with their patients efficiently. Therefore, many health care centre have started using online appointment systems. Such systems ensure efficiency and effectiveness.

Online Booking System is an innovative web-based platform designed to manage and facilitate processes related to patient registration and waiting room management. patient online booking system has been proven to successfully decrease patient queuing times and increase efficiency across all our sites. Patients access a system that is optimized for use on touch screens and a variety of devices including computers, tablets, phones and kiosks. Patients follow a step by step process to self- register into a service or hospital. A duplication checker ensures that only one record is held per patient containing all demographic information. Unique IDs are generated and used in connection with NHS, hospital and local patient identifiers to locate patient records quickly.

\section{SYSTEM OVERVIEW}

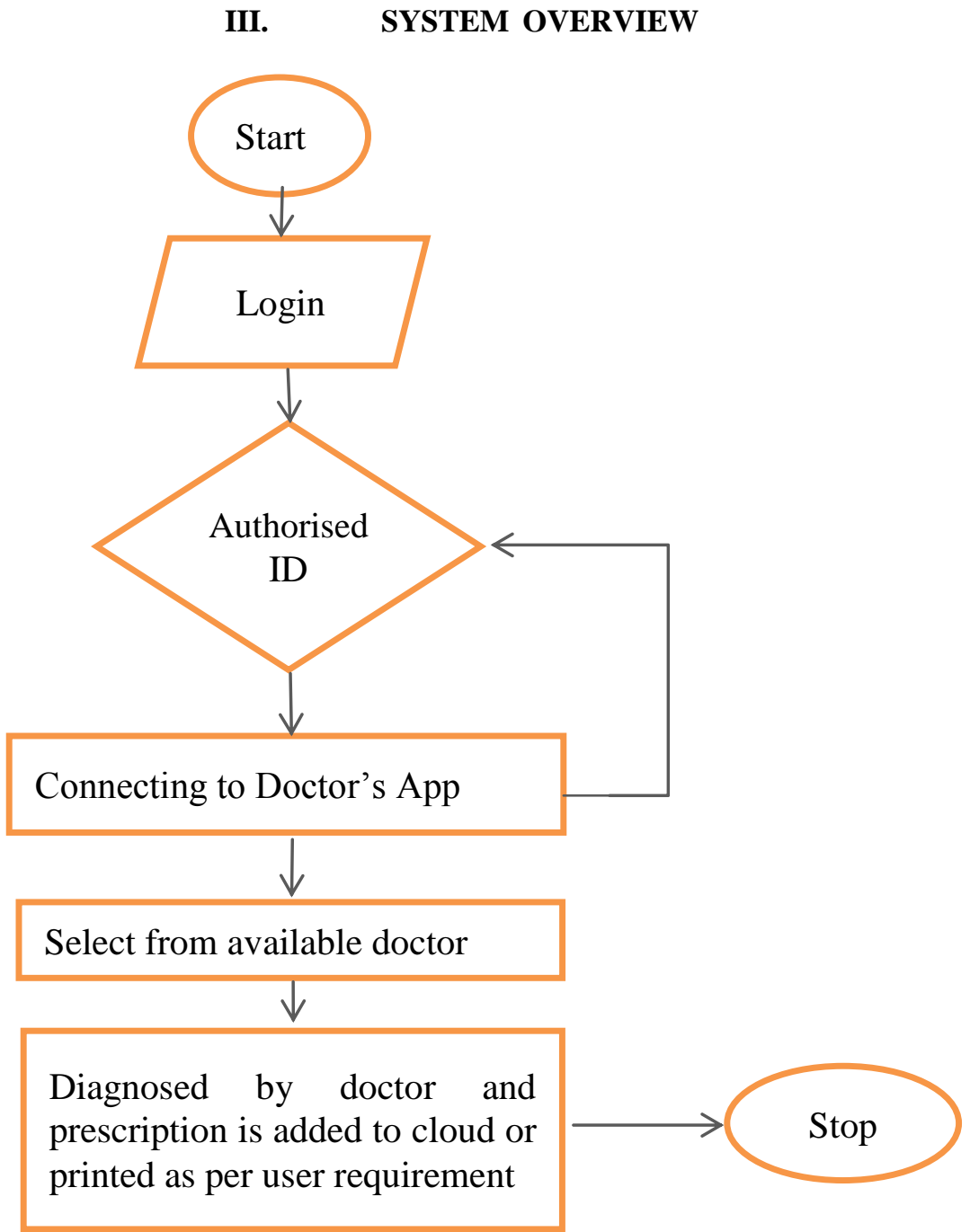


Vol. 8, Issue 4, April 2019

The proposed system is kiosk which is similar to a ATM kiosk with minimum requirements. Here patient need to be register their physical information through an Android platform or through kiosk, which is also applicable to doctors by then they will get their registration id and a pin and those who are illiterate to use this Android platform can enroll themselves using their bio-metrics this is the first step you need to be done before diagnosing Then at time of diagnosing user need to sign using their Registration id or bio-metrics information as per their interest, then user need to select which doctor they need, then they will be connected to available doctor and all the primary test can be done with in the kiosk and doctors prescription can be either be printed or get mailed or can be forwarded to pharmacy as per users interest, in meanwhile at the doctor side he/she will get inference or prediction using machine learning, the final reports are will be stored inside the cloud.

\section{METHODOLOGY}

A Crucial phase in the System life cycle involves Successful implementation of new system design, Implementation involves Development of android app and also involves setting the relationship between Doctors , Giving Training to Doctors and Users to minimize resistance to change, Phases Of Implementation Involves,Development android apps,Setting relationship between Doctors and Users, Training Doctors, pharmacist, lab technicians and patients

4.1:Preliminary Considerations: Before the actual design of the project work, specific deliberate choices in selection of appropriate implementation platforms and hardware components mare mase. Priority was given to low cost availability, reliability, flexibility and simplicity in all these selections.

4.2: System Requirements: The Android application for the registration need to be build using the Android Studio, The software in the kiosk is build using Java as background and is build on Windows Embedded ,The machine learning section which is done on cloud platform uses the python programming and at the doctors end he/she will need to install the Dr.Kiosk's app which is build on Android.

Google Fire Base Console is a Database, which is used to store real-time data

TokBox for peer to peer video call session

Heroku Cloud for Server to handle session generation requests.

Android Studio, Java\& XML to develop android Applicatio::

PHP for TokBox Server Implementation

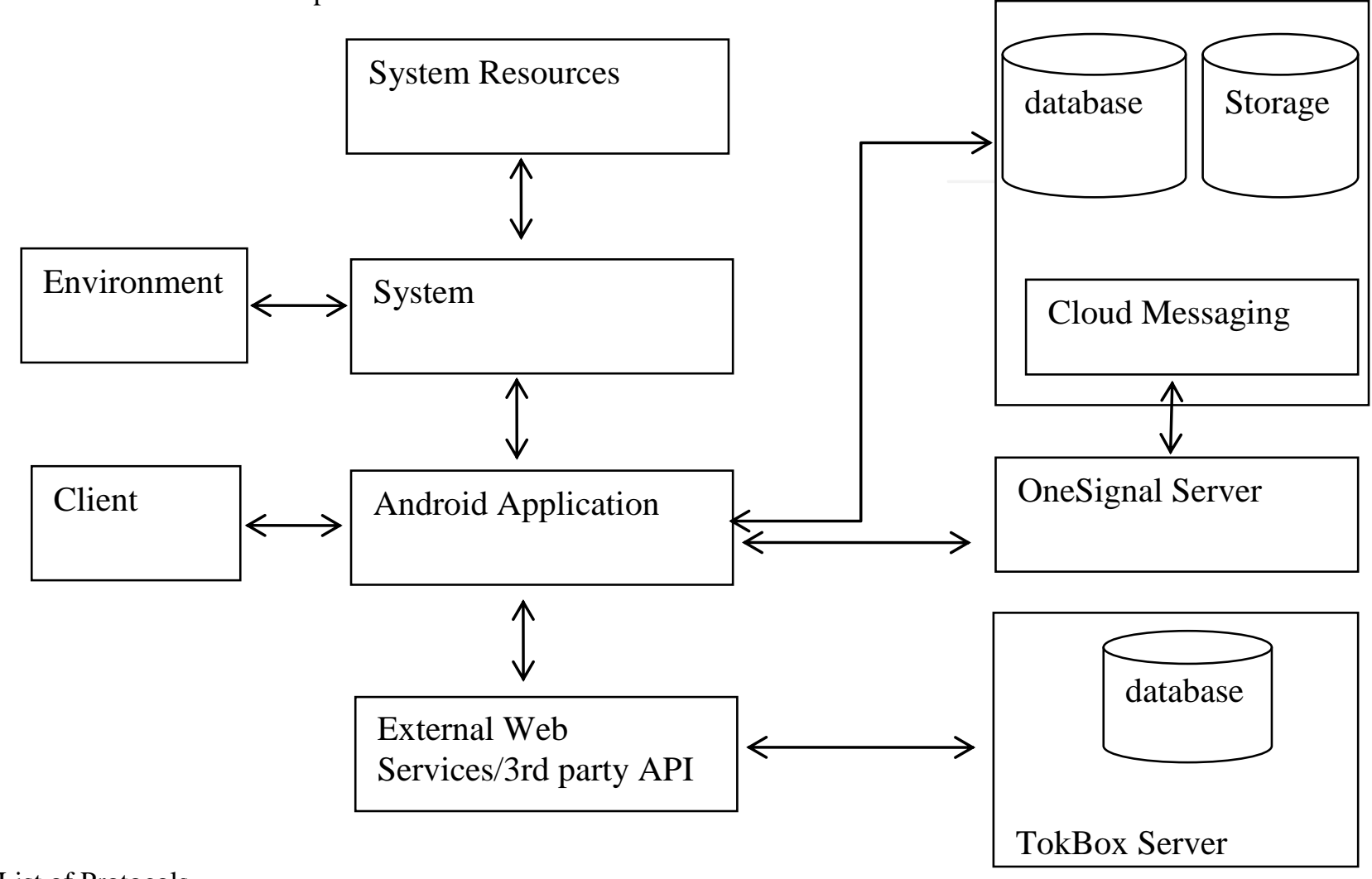

List of Protocols

HTTP Client Server Communication 
Vol. 8, Issue 4, April 2019

REST for REst based interactions to call services

RTP To manage realtime transmission of multimedia data over networked services.

Peer to Peer for web RTC to Manage transmission data between two clients.

Fire Database to store and sync data in realtime with NoSQL cloud databse

Fire storage Tupload and share user generated contents such as images and video

OpenTok client SDK to create OpenTok sessions, tokens and work with OpenTok archiving

Volley HTTP library for scheduling concurrent network requests

OneSignal Implementing reliable reliable push notification Services on our actions

\section{IMPLEMENTATION}

The system contains various modules such as Heart beat sensor, Pressure sensor, Touchscreen, webpage, Android Application, Arduino, GSM module, notification are discussed in detail below:

1:Heart beat Sensor: Heartbeat sensor measyres the change in volume of blood through any organ of the body which causes a change in he light intensity through that organ.

2: Pressure sensor: Non-invsive sensor designed to measure human blood pressure. it measures systolic, doastolic and arterial pressure

3: TouchScreen: 7inch Screen which help the user to interact with Kiosk, And also it will give Guidens to the user 4: Webpage: The camera is accessed to the webpage and also the light, fan, door lock, alarm can be controlled with the webpage. The owner can directly login and interact with the webpage and the control unit provided. The images captured and the videos recorded will be directly streamed on user pre-decided webpage on smart phone.

5: Android Application: Android Application which is completely build for Docters

6: Arsuino: Arduino Uno is a microcontroller board based on the ATmega328P (datasheet). It has 14 digital input/output pins (of which 6 can be used as PWM outputs), 6 analog inputs, a $16 \mathrm{MHz}$ quartz crystal, a USB connection, a power jack, an ICSP header and a reset button.

8: GSM module: GSM was designed with a moderate level of service security. The system was designed to authenticate the subscriber using a pre-shared key and challenge-response. Communications between the subscriber and the base station can be

\section{RESULT AND DISCUSSION}

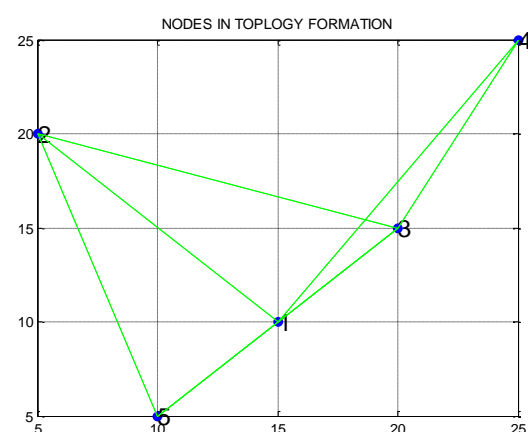

Fig.1. Ad Hoc Network of 5 Nodes

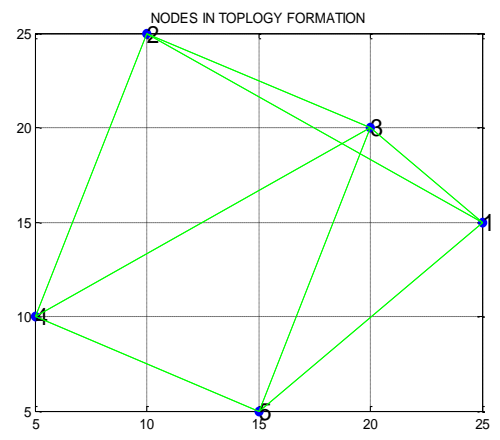

Fig. 3. Ad Hoc Network of 5 Nodes

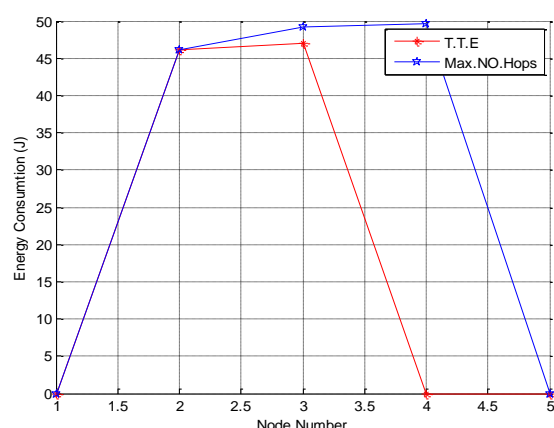

Fig. 2. Energy Consumption by Each Node

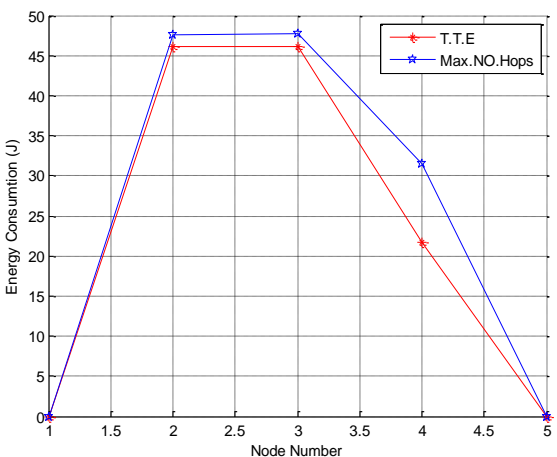

Fig 4. Energy Consumption by Each Node 
Vol. 8, Issue 4, April 2019

The final outcome of this project is a prototype for a simple home security system which can control home appliances with a touch of finger and is equally capable of monitoring with live stream video feed of the webpage. On the front end, there is a microcontroller to control the home appliances and PCB board to which a number of home appliances are connected. Then on the backend, there is a raspberry pi which has been programmed to control the status of GPIO pins and thereby controlling the appliances and also capture video using camera and stream it live to your webpage created.

\section{CONCLUSION AND FUTURE WORK}

Challenge is to cater to the deserving under served community of Tier 4 locations and below which need due attention. Public health services have done poorly even along the income distribution dimension. According to a study, the poorest $20 \%$ of the population captures only $10 \%$ of the public health subsidy as compared to $30 \%$ by the richest $20 \%$. The share in the subsidy rises monotonically as we move from the bottom $20 \%$. The discrepancy and poor implementation of budget allocation have left a stark gap in rural and urban health care services. With India ascending the health care services on the tertiary level and becoming the new face of global medical tourism, it dampens the zeal to notice the staggering figure of major gamut being unable to access affordable primary health care services which are necessary for the development of the nation.

The DR.KIOSK is virtual Doctor Kiosk where a person could get diagnosed with virtual presence of a Doctor with AI assistance, which means same quality treatment available in hospital will be available inside a cubicle that too without having a physical presence of your doctor with intelligent system for reducing diagnosing errors, DR KIOSK is similar to Auto Teller Machine where you can meet your Doctor Online through this kiosk. Dr.Kiosk is thus all set to revolutionize the health-care sector with its state of the art UI/UX design, functionality and thus act as a benchmark for other online health-care consultancy services. In today's there are many apps available in markets for fixing appointment of patients with doctor through online but they does not meet all the requirement of the patients. So we are developing this kiosk so that the patient doesn't need to carry all that records to doctor who is $30 \mathrm{~km}$ or more far apart, he/she can also meet her doctor in the nearest junction from his. her home ,the Dr.Kiosk bring the power of Data mining , $\mathrm{AI}$ and Machine learning to predict the prescription and the diseases that could make the doctor to look into and thus reducing the medical negligence, by keeping the all record online we could also say that it will reduce the medical negligence due to loss of medical record.

In Future by using the details of analysis of medical data we could power a Intelligent system that doesn't make any errors in the while predicting the disease or never commit a medical negligence so that we could provide medicine and diagnosing to the underprivileged people of the society without a penny

\section{REFERENCES}

[1]. Anjum Asma and Gihan Nagib,'Energy Efficient Routing Algorithms for Mobile Ad Hoc Networks-A Survey', International Journal of Emerging Trends \& Technology in computer Science, Vol.3, Issue 1, pp. 218-223, 2012.

[2]. Hong-ryeol Gil1, Joon Yoo1 and Jong-won Lee2 ,'An On-demand Energy-efficient Routing Algorithm for Wireless Ad hoc Networks', Proceedings of the $2^{\text {nd }}$ International Conference on Human. Society and Internet HSI'03, pp. 302-311, 2003.

[3]. S.K. Dhurandher, S. Misra, M.S. Obaidat, V. Basal, P. Singh and V. Punia,'An Energy-Efficient OnDemand Routing algorithm for Mobile AdHoc Networks', $15^{\text {th }}$ International conference on Electronics, Circuits and Systems, pp. 958-9618, 2008.

[4]. DilipKumar S. M. and Vijaya Kumar B. P. ,'Energy-Aware Multicast Routing in MANETs: A Genetic Algorithm Approach', International Journal ofComputer Science and Information Security (IJCSIS), Vol. 2,2009.

[5]. AlGabri Malek, Chunlin LI, Z. Yang, Naji Hasan.A.H and X.Zhang,' Improved the Energy of Ad hoc On- Demand Distance Vector Routing Protocol', International Conference on Future Computer Supported Education, Published by Elsevier, IERI, pp. 355-361, 2012.

[6]. D.Shama and A.kush,'GPS Enabled E Energy Efficient Routing for Manet', International Journal of Computer Networks (IJCN), Vol.3, Issue 3, pp. 159-166, 2011.

[7]. Shilpa jain and Sourabh jain, 'Energy Efficient Maximum Lifetime Ad-Hoc Routing (EEMLAR)', international Journal of Computer Networks and Wireless Communications, Vol.2, Issue 4, pp. 450-455, 2012.

[8]. Vadivel, R and V. Murali Bhaskaran,'Energy Efficient with Secured Reliable Routing Protocol (EESRRP) for Mobile Ad-Hoc Networks', Procedia Technology 4,pp. 703- 707, 2012.

[9]. Nobuo Ezaki, Marius Bulacu Lambert, Schomaker, "Text Detection from Natural Scene Images: Towards a System for Visually Impaired Persons", Proc. of 17th Int. Conf. on Pattern Recognition (ICPR), IEEE Computer Society, pp. 683-686, vol. II, 2004

[10]. Mr. Rajesh H. Davda1, Mr. Noor Mohammed, “ Text Detection, Removal and Region Filling Using Image Inpainting”, International Journal of Futuristic Science Engineering and Technology, vol. 1 Issue 2, ISSN 2320 - 4486, 2013

[11]. Uday Modha, Preeti Dave, “Image Inpainting-Automatic Detection and Removal of Text From Images”, International Journal of Engineering Research and Applications (IJERA), ISSN: 2248-9622 Vol. 2, Issue 2, 2012.

[12]. Muthukumar S, Dr.Krishnan .N, Pasupathi.P, Deepa. S, "Analysis of Image Inpainting Techniques with Exemplar, Poisson, Successive Elimination and 8 Pixel Neighborhood Methods", International Journal of Computer Applications (0975 - 8887), Volume 9, No.11, 2010 